\title{
EL EMPRENDEDOR DOMINICANO: IDENTIFICACIÓN DE SUS RASGOS DE PERSONALIDAD Y COMPETENCIAS POR MEDIO DE ANÁLISIS DE CONTENIDOS
}

\author{
Fernando Barrero Rivera ${ }^{a}$, Eric Moreno ${ }^{\mathrm{b}}$ y JaVier Villamizar \\ FERNÁNDEZ
}

Recibido: Julio, 2019 • Aprobado: Septiembre, 2019

Cómo citar: Barrero Rivera, F., Moreno, E., \& Villamizar Fernández, J. (2019). El emprendedor dominicano: identificación de sus rasgos de personalidad y competencias por medio de análisis de contenidos. Ciencia, Economía y Negocios, 3(1), 5-36. Doi: https://doi.org/10.22206/ceyn.2019.v3i1.pp5-36

\section{Resumen}

El propósito de esta investigación es identificar los rasgos de personalidad y las competencias de un grupo de emprendedores dominicanos, de manera que el ecosistema emprendedor, incluyendo la población de empresarios, estudiantes, nuevos emprendedores, el gobierno, el sector académico y las ONG involucradas, generen espacios y sistemas que fortalezcan las competencias emprendedoras y el entorno emprendedor, en función de mejorar la calidad de vida poblacional, así como contribuir a la sostenibilidad social y económica de la nación. El estudio se centra en mostrar los resultados de la información recopilada por estudiantes del MBA de Barna Management School, donde se caracteriza a una muestra de emprendedores dominicanos por medio del análisis de contenidos. Los resultados señalan que los dos rasgos de personalidad comunes entre los emprendedores consultados son la pasión y la aceptación del riesgo, los cuales son determinantes en el actuar de todo el que emprende. Como competencias relevantes se encuentran las involucradas en la "Orientación Emprendedora", más especificamente, la creatividad (incluye la generación de ideas) y la percepción de oportunidades (proactividad).

Palabras clave: ecosistema emprendedor; competencias; entorno emprendedor; rasgos de personalidad; análisis de contenido; orientación emprendedora; ONGs.

Códigos JEL: M12, M13, M14.

\footnotetext{
aBarrero Rivera, A.: Barna Management School. Dirección: Av. John F. Kennedy No. 34, Naco. Santo Domingo, República Dominicana. T: +809 683 4461, correo-e: Fernando.barrero@barna.edu.do

bMoreno, E.: Barna Management School. Dirección: Av. John F. Kennedy No. 34, Naco. Santo Domingo, República Dominicana. T: +809 223 4409, correo-e: Eric.moreno@barna.edu.do

'Villamizar Fernández, J.: Barna Management School. Dirección: Cra. 36 \#8 oeste 14, Cali - Valle, Colombia. T: +57 320 3498979, correo-e: javierfvillamizarf@yahoo.com

${ }^{\mathrm{d} C o n c e p t o ~ a m p l i a m e n t e ~ d e s c r i t o ~ p o r ~ A n l e s i n y a ~ y ~ B o n u e d i ~(2015) . ~}$
} 


\title{
THE DOMINICAN ENTREPRENEUR: IDENTIFICATION OF HIS PERSONALITY TRAYS AND COMPETENCES THROUGH CONTENT
} ANALYSIS

\author{
Fernando Barrero Rivera, Eric Moreno y Javier Villamizar \\ FERNÁNDEZ
}

Received: july, 2019• Approved: September, 2019

\begin{abstract}
The purpose of this research is to identify the personality traits and competencies of a group of Dominican entrepreneurs, so that the entrepreneurial ecosystem, including the population of entrepreneurs, students, new entrepreneurs, the government, the academic sector and involved NGOs, generate spaces and systems that strengthen entrepreneurial skills and the entrepreneurial environment in terms of improving the quality of life of the population, as well as contributing to the social and economic sustainability of the nation. The study focuses on showing the results of the information gathered by students of the MBA of Barna Management School, where a sample of Dominican entrepreneurs is characterized through content analysis. The results indicate that the two personality traits in common among the entrepreneurs consulted are the passion and acceptance of risk, which are decisive in the actions of everyone who undertakes. As relevant competences are those involved in the Entrepreneurial Orientation, more specifically, creativity (including the generation of ideas) and the perception of opportunities (proactivity).
\end{abstract}

Keywords: Entrepreneurial ecosystem; competencies; entrepreneurial environment; personality traits; content analysis; entrepreneurial orientation; $N G O s$.

JEL Codes: M12, M13, M14. 


\section{Introducción}

\section{Ecosistema emprendedor}

Todo grupo interconectado de personas emprendedoras (potenciales, nacientes y existentes), de agentes financiadores (empresas, venture capitalists, business angels, bancos), y de organizaciones promotoras (universidades, agencias del sector público) asociadas a la diversidad de iniciativas emprendedoras y con una orientación de generar valor en el territorio ${ }^{a}$ se le conoce como ecosistema emprendedor; en síntesis, el ecosistema emprendedor se compone del entorno emprendedor y de los emprendedores.

Otro concepto, recientemente publicado, es el considerado por Bruns, Bosma, Sanders y Schram (2017) quienes definen el ecosistema emprendedor como un conjunto multidimensional de factores interactivos que moderan el efecto de la actividad empresarial en el crecimiento económico. En la investigación desarrollada por estos investigadores, encontraron que existe una contribución homogénea del espíritu empresarial al crecimiento económico de 107 regiones europeas en dieciseis Estados miembros de la Unión Europea (Bruns et al., 2017).

Los ecosistemas de emprendimiento emergen como un compromiso para fomentar el espíritu empresarial y el desarrollo económico en un contexto regional (Cagica, 2017). El sector público de Lisboa lideró la optimización de un ecosistema centrado en mejorar su infraestructura, tecnología e innovación. En la actualidad, Lisboa ofrece infraestructuras (como incubadoras de empresas, aceleradores, centros de investigación, universidades, etc.) y un entorno empresarial marcado por tendencias internacionales, creatividad y disponibilidad de recursos humanos calificados. Este fenómeno ha permitido que Portugal se encuentre en el puesto 9 en accesibilidad al talento humano, de 60 países, por otro lado, su ubica en el top 5 del mejor desempeño de empresas nacientes de Europa, también se posiciona en el lugar 31 del Índice de Innovación Global, frente a 127 países del mundo. Lo anterior ha sido fruto de un esfuerzo mancomunado entre el gobierno central y los sectores económicos,

aPara más detalles, consultar a Mason y Brown (2014); Reynolds (2005); Veciana y Urbano (2008), Gnyawali y Fogel (1994); Welter y Smallbone (2011); Aparicio, Urbano y Gómez (2018). 
que se propusieron generar sistemas integrados de conectividad y acceso a información actualizada de mercados nacionales y mundiales, así como a la implementación de incubadoras y aceleradoras de negocios. Es así como, desde la formulación de las políticas nacionales, Portugal goza de un ecosistema diseńado para competir. El reporte de "Mapping Entrepreneurship Ecosystems" (Cuppen, 2018), expone cómo las redes, el sistema educativo, el apoyo del gobierno, el acceso al capital y la experticia son dimensiones sobre las que construyen una infraestructura de entorno emprendedor.

\section{Rasgos de personalidad}

Según un estudio reciente de la Harvard Business School (Pekkala, 2017), donde se revisó una extensa literatura desde el año 2000 sobre los rasgos de personalidad de los emprendedores, se encontró que son la autoeficacia, la capacidad de innovación (identificar oportunidades), el control y la necesidad de logro las características que definen el perfil emprendedor. Otros estudios mencionaron la confianza en sí mismo (Barron y Harrington, 1981), la apertura a la experiencia (George y Zhou, 2001), la proactividad (Seibert, 2001), la necesidad de logro y la aceptación del riesgo (Rauch \& Frese, 2007), en gran parte corroborados en una amplia investigación desarrollada por Viinikainen, Heineck, Böckerman, Hintsanen, Raitakari y Pehkonen (2016) como rasgos de la personalidad de emprendedores.

\section{Competencias emprendedoras}

Por otro lado, las competencias involucradas en la Orientación Emprendedora ${ }^{\mathrm{b}}$ se relacionan con la creatividad (incluye la generación de ideas), la percepción de oportunidades (proactividad), y la capacidad de tomar riesgos. En este sentido, Davis, Hall y Mayer (2015) corroboraron los hallazgos de Brandstätter (2010), quien investigó sobre aspectos de la personalidad del espíritu emprendedor, con información proveniente de cinco meta-análisis. Por ejemplo, encontró algunos rasgos como pre-

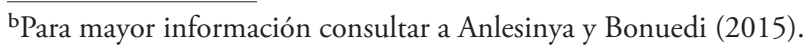


paración para la innovación, personalidad proactiva, autoeficacia generalizada, tolerancia al estrés, necesidad de autonomía, y necesidad de control (Davis et al., 2015). También halló correlaciones significativas de la creación de empresas con el éxito empresarial, así como la propensión al riesgo, que apoya la base de negocios, pero no necesariamente el éxito empresarial. Adicionalmente, anotó que la motivación de logro es favorable tanto para la fundación empresarial como para el éxito empresarial (Brandstätter, 2010).

$\mathrm{Al}$ considerar las competencias emprendedoras, una aproximación adicional corresponde a algunos autores que se han puesto de acuerdo en que una iniciativa exitosa depende en gran parte de los intereses de los emprendedores (Schmitt, 2004). Adicionalmente, algunos expertos (Hills y Hultman, 2006; Hamel, 2000, citados en Morrish, Deacon y Miles, 2010), han identificado ciertas características de las empresas emprendedoras que prolongan la creatividad de la siguiente manera:

- Acercamiento flexible y personalizado al mercado.

- Reacciones rápidas a los cambios en la preferencia del cliente.

- La planificación ocurre en pasos incrementales cortos (tácticos).

- La investigación de mercado formal es inusual.

- Confianza en la intuición y la experiencia.

- Esfuerzo por liderar a los clientes.

- Creación de valor a través de relaciones y alianzas.

- La visión y la estrategia son impulsadas por el éxito táctico.

- Innovación en productos, servicios y estrategias.

En las competencias, Davis et al. (2015) consideran factores importantes tales como el enfoque en el futuro, la generación de ideas, la ejecución, la autoconfianza, el optimismo, la persistencia, la sensibilidad interpersonal, según lo que se describe a continuación:

- Enfoque en el futuro: la capacidad de pensar más allá de la situación inmediata y planificar para el futuro (por ejemplo: "me concentro en el largo plazo"). 
- Generación de ideas: la capacidad de generar ideas múltiples y novedosas y de encontrar diversos enfoques para lograr objetivos (por ejemplo: "algunas veces las ideas solo emergen de mí").

- Ejecución: la capacidad de convertir ideas en planes accionables; la habilidad de implementar ideas.

- Confianza en sí mismo: una creencia general en la capacidad de uno de aprovechar habilidades y talentos para alcanzar objetivos importantes.

- Optimismo: la capacidad de mantener una actitud generalmente positiva sobre diversos aspectos de la vida.

- Persistencia: la capacidad de recuperarse rápidamente de la desilusión y permanecer persistente frente a los reveses.

- Sensibilidad interpersonal: un alto nivel de sensibilidad y preocupación por el bienestar de aquellos con los que uno trabaja.

$\mathrm{Al}$ respecto, la Organización para la Cooperación y el Desarrollo Económicos (OCDE) (2018) en su artículo "Desarrollando las competencias de emprendimiento", identifican a los emprendedores como personas que combinan creatividad, sentido de iniciativa, resolución de problemas, capacidad para reunir recursos, así como también cuentan con conocimiento financiero y tecnológico. El informe menciona que "éstas competencias permiten a los emprendedores provocar y adaptarse al cambio, las cuales se pueden desarrollar a través de la educación y la capacitación en emprendimiento, promoviendo una mentalidad y comportamiento empresarial". (OECD, 2018, p. 3).

Cuando la definición de espíritu empresarial se expande para abarcar cualquier tipo de valor, ya sea social, cultural, ambiental o económico, el espectro de conocimientos, habilidades y actitudes que conforman el núcleo de la competencia se amplía e incluye a características tales como creatividad, identificación de oportunidades, autoeficacia, confianza en sí mismo, comunicación, liderazgo, toma de decisiones, innovación, responsabilidad, colaboración, generación de ideas, resolución de problemas, autonomía, negociación y trabajo en red (Komarkova, I., Gagliardi, D., Konrads, J., Collado, A., 2015). El inventario de competencias incluye las relacionadas con el conocimiento, habilidades y actitudes. Sobre la base 
de la revisión de la literatura académica, la lista se puede agrupar en tres áreas conceptuales principales que abordan: i) competencias operativas y contextuales: es decir, aquellas que se refieren al conocimiento y las habilidades sobre el espíritu empresarial; ii) competencias emprendedoras: las relacionadas con la identificación, exploración, evaluación y explotación de oportunidades de creación de valor; y iii) competencias conceptuales y de relación: actitudes y habilidades orientadas a la acción que aprovechan tanto a los sujetos emprendedores como a su red y se refieren, principalmente, a aquellos aspectos de la competencia que se desarrollan a través del espíritu empresarial (Komarkova et al., 2015, p. 8).

\section{Pensamiento efectual}

En las últimas dos décadas, un importante número de investigaciones ha encontrado que los emprendedores exitosos cuentan con un modelo de pensamiento efectual, el cual logra vincular en parte los rasgos de personalidad y las competencias emprendedoras. Este concepto fue introducido en 2001 por la Dra. Saras Sarasvathy a partir de investigaciones con emprendedores en la Darden School of Business de la Universidad de Virginia. Este tipo de pensamiento ha sido descrito con las siguientes características de los emprendedores (Sarasvathy, 2003):

- Presentan un pensamiento en términos de control, no de incertidumbre.

- Mantienen una visión flexible, asumiendo que se alcanzará la meta por aproximaciones sucesivas.

- Consiguen los primeros clientes y socios comprometidos, que luego serán quienes suministren nuevos medios y nuevas metas.

- Reducen el riesgo de la empresa y obtienen clientes e ingresos de la forma más rápida.

- Aprovechan todo lo que se descubre en el proceso que pueda ser de utilidad.

- Arriesgan cuando las pérdidas son asumibles en el caso de fracaso y distribuyen el riesgo a los demás. 
- Su centro de acción se mantiene en la búsqueda de oportunidades de mercado, viendo aquello que no está satisfecho y generan una nueva circunstancia para capitalizar.

- Parten de los medios y recursos disponibles para crear soluciones y avanzar.

La investigadora Sarasvathy relaciona algunos principios que identifican la experiencia de un emprendimiento. Los emprendedores expertos invierten los principales criterios de toma de decisiones en las teorías recibidas y las prácticas de gestión convencionales (Sarasvathy, 2003), por medio de los siguientes principios:

- El principio del edredón de retazos. Este es un principio de acción impulsado por los medios (en oposición a los objetivos). El énfasis aquí es crear algo nuevo con los medios existentes, para descubrir nuevas formas de lograr los objetivos.

- El principio de pérdida accesible. Este principio prescribe comprometerse por adelantado con lo que uno está dispuesto a perder en lugar de invertir en cálculos sobre los rendimientos esperados del proyecto.

- El principio del pájaro en mano. Este principio implica negociar con todas las partes interesadas que estén dispuestas a asumir compromisos reales con el proyecto, sin preocuparse por los costos de oportunidad ni llevar a cabo análisis competitivos elaborados.

- El principio de la limonada. Este principio sugiere reconocer y apropiarse de la contingencia mediante las sorpresas en lugar de tratar de evitarlas, superarlas o adaptarlas.

- El principio del piloto en el avión. Este principio insta a depender y trabajar con el talento humano como el principal conductor de oportunidades, en lugar de limitar los esfuerzos empresariales para explotar factores exógenos tales como trayectorias tecnológicas y tendencias socioeconómicas.

Cada uno de los cinco principios anteriores incorpora técnicas de control no predictivo, es decir, reducir el uso de estrategias predictivas para controlar situaciones inciertas. Juntos, estos principios apuntan a 
una lógica de acción llamada efectual. De toda la revisión de literatura mencionada anteriormente, se puede deducir que las competencias para identificar en un emprendedor se asocian en parte con los principios de pensamiento efectual propuestos por Sarasvathy (2003), así como el listado de características relacionadas por Davis et al., (2015).

\section{Método}

La información fue recolectada en 99 entrevistas con emprendedores dominicanos consultados entre los años 2015 y 2018 (ver detalle en el anexo 1), por medio de los estudiantes de las diferentes cohortes del MBA de Barna Management School. La data, consistente en 186 minutos de grabación, cuya transcripción correspondió a más de 220.000 palabras, se examinó por medio de la técnica de análisis de contenidos, con ayuda del paquete Atlas.ti (desarrollo de integración de operaciones semánticas de información antropológica).

El perfil de la muestra consistió en su mayoría hombres, con empresas que se encontraron en 32 sectores diferentes (ver detalle en anexo 2).

\section{Resultados}

\section{Rasgos de personalidad del emprendedor dominicano}

De acuerdo con los resultados de la presente investigación, los emprendedores dominicanos seleccionados se pueden describir como profesionales con dos rasgos de personalidad: pasión y aceptación del riesgo (Gráfico 1), los cuales son determinantes en el actuar de todo el que emprende (Davis et al., 2015).

De manera adicional, algunos de los emprendedores dominicanos entrevistados son temerarios, osados, tercos, arriesgados, apasionados por su empresa, prácticos y creativos. Es importante mencionar que los dominicanos emprendedores deciden abrir nuevos negocios en su país, usualmente en mercados competidos, pero sin temores al fracaso (ver detalle en anexo 3). 
Gráfico 1. Porcentaje de percepciones que se asocian a los rasGOS DE PERSONALIDAD

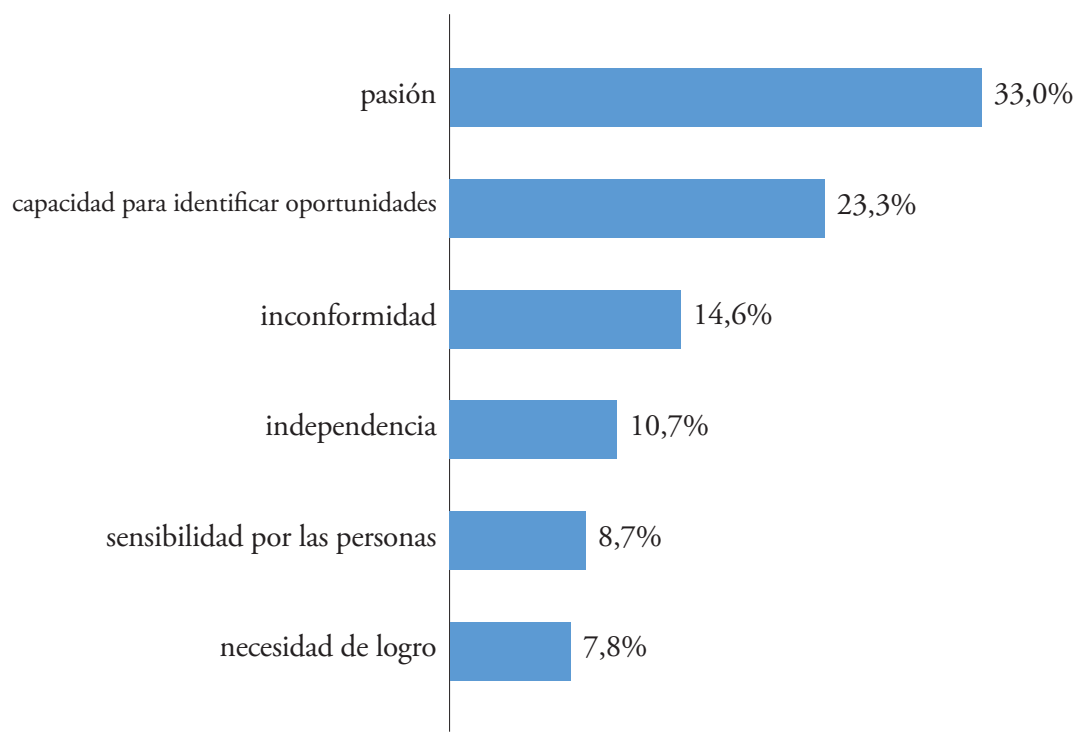

Fuente: elaboración de los autores, el valor porcentual es obtenido sobre el total de la muestra (99 entrevistas).

Los rasgos, referidos a esos componentes de la personalidad hallados en los emprendedores dominicanos, se validan a partir de una investigación desarrollada por Mark Davis y sus colaboradores (Davis et al., 2015), quienes propusieron los siguientes rasgos en los emprendedores: independencia, estructura limitada, no conformidad, aceptar el riesgo, orientar a la acción, pasión y necesidad de logro. De la misma manera, la identificación de oportunidades y la necesidad de logro son otros rasgos que se corroboran con el amplio estudio de Harvard Business School (Pekkala, 2017).

\section{Competencias del emprendedor dominicano}

Con base en la clasificación abordada al inicio del presente texto, la figura 1 presenta las competencias de los emprendedores entrevistados. 
Operacional y contextual

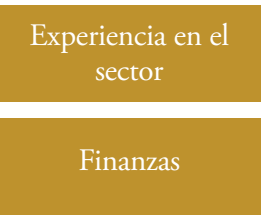

Planeación

Investigación empírica
Emprendimiento
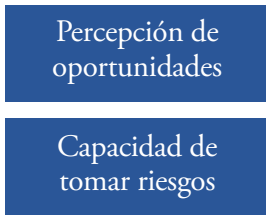

Generación de ideas

Innovación
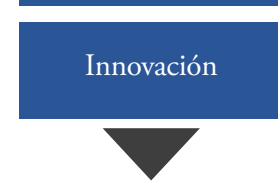

Generación de valor
Conceptual y de relación

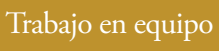

Perseverancia

Auto confianza

Formación de redes

Figura 1. Competencias de los emprendedores dominicanos consultados

Fuente: elaboración de los autores, con base en el modelo conceptual de Pekkala (2017).

A continuación, se detalla la información de cada uno de los grupos de competencia:

\section{Competencias de orientación emprendedora}

Las competencias que se pueden clasificar dentro del grupo de Orientación Emprendedora y que más caracterizaron al grupo de emprendedores entrevistados son la generación de ideas y la aceptación del riesgo, fundamentales para asumir el reto de emprender (Gráfico 2).

Implícitamente, la totalidad de los emprendedores investigados identificaron oportunidades a partir de su proceso de observación, o al encontrarse con la opción de continuar con el negocio de su familia. Asimismo, algunos manifestaron ser intuitivos, investigadores empíricos, quienes reinvierten en su negocio y trabajan más de 12 horas al día (ver detalle en anexo 4).

Al tener en cuenta la reciente información del Global Entrepreneurship Monitor (Barna Management School y Ministerio de Industria, Comercio y Mypimes, 2019), tan solo el 20 \% de la muestra de empresarios (y emprendedores) consultados, manifestaron que el miedo al fracaso sería 
un obstáculo para poner en marcha un negocio, de manera que, para el dominicano emprendedor, aceptar el riesgo logra vencer las posibles barreras del miedo al fracaso.

Gráfico 2. Porcentaje de percepciones asociadas a competencias de Orientación Emprendedora

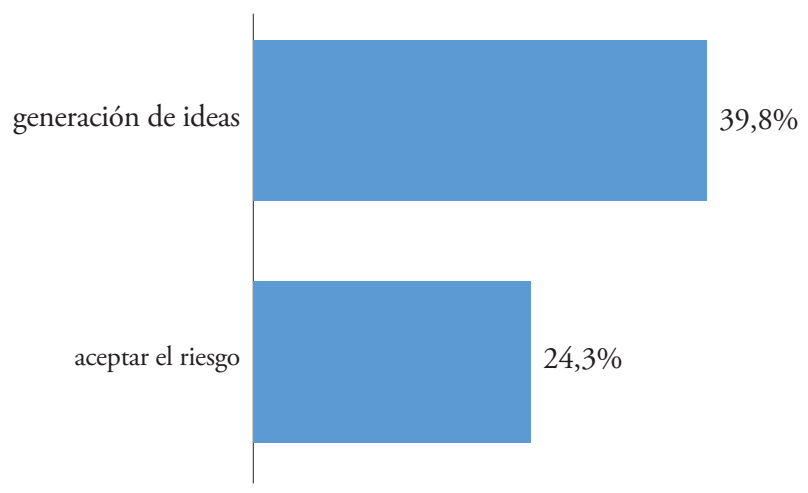

Fuente: elaboración de los autores, el valor porcentual es obtenido sobre el total de la muestra.

Estos hallazgos corroboran los resultados obtenidos en las investigaciones de Tiago, Faria y Couto (2015), quienes identificaron que los emprendedores piensan y actúan evaluando los beneficios potencialesa partir de oportunidades percibida compensando los riesgos y amenazas posibles. Este comportamiento es el determinante de las intenciones emprendedoras.

Por otra parte, si se asocia la creatividad al proceso de generación de ideas, el más reciente reporte de la OECD (2015) afirma que una de las competencias de los emprendedores es la creatividad, de manera que se corroboran los resultados de la presente investigación.

\section{Competencias conceptuales y de relación}

En estas se incluyen el trabajo en equipo, autonomía, autoconfianza y perseverancia; los emprendedores de la muestra seleccionada, al respecto, presentan altos niveles de persistencia, inclusive, algunos manifestaron que, 
a pesar de las circunstancias negativas, su comportamiento pasó los límites de la terquedad (Gráfico 3). El trabajo en equipo con esfuerzo, hasta en jornadas diarias mayores a 12 horas, fue el común en la mayor parte de los emprendedores. Todo lo anterior se alimentó continuamente de una gran autoconfianza y optimismo muy marcado (ver detalle en anexo 4).

Para validar lo anterior, una reciente investigación (Cruz-Ros, Mas-Tur y Garzón, 2017) demostró que la forma en que los emprendedores se ven a sí mismos, su confianza en sí mismos, su forma de trabajar y sus percepciones en general, los hace sentir más seguros cuando se enfrentan a nuevos desafíos que cuando no se enfrentan a ellos. Estos factores se pueden analizar por separado, pero algunos autores como Monllor y Altay (2016), agrupan estos factores bajo el concepto general de autoeficacia. De una forma u otra, la literatura contiene evidencia de una relación clara y positiva entre estas características y la intención de iniciar un negocio. De la misma manera, los exhaustivos estudios de Brandstätter (2010) y Davis et al., (2015) afirman que la generación de ideas, el optimismo y la persistencia, corresponden a competencias de quienes emprenden, validando los hallazgos del presente estudio.

Gráfico 3. Porcentaje de percepciones asociadas a competencias CONCEPTUALES Y DE RELACIÓN

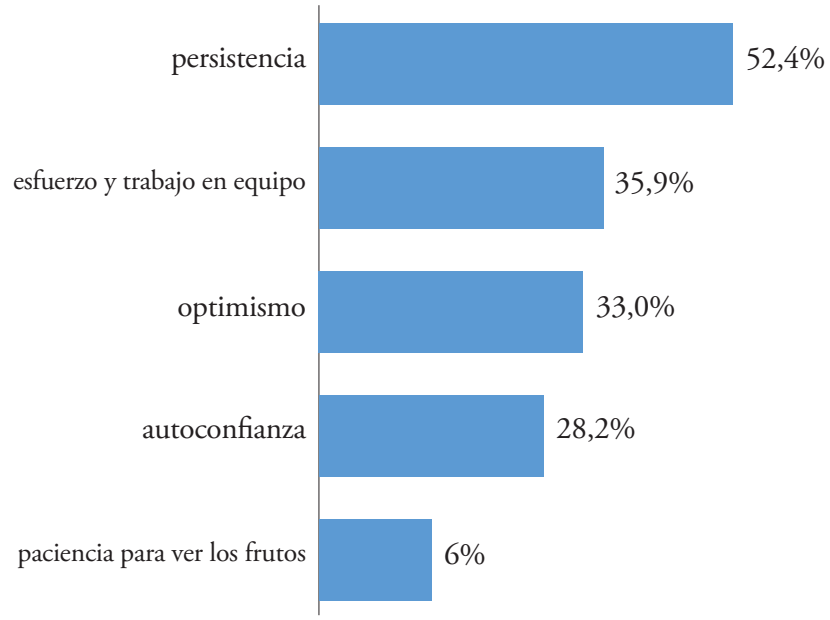

Fuente: elaboración de los autores, el valor porcentual es obtenido sobre el total de la muestra. 


\section{Competencias operacionales y contextuales}

En este grupo de competencias, una cuarta parte de los entrevistados desarrollan técnicas predictivas e investigativas, vitales en la etapa de planeación (ver gráfico 4). Otra cuarta parte, recurre a procesos de mercadeo estratégico y alianzas, como parte de su planeación.

Para los emprendedores dominicanos entrevistados, la investigación de mercados ha sido elaborada dentro de un modelo informal, no científico, de carácter exploratorio, para validar el nivel de aceptación del concepto de negocio. En la mayoría de las empresas nuevas, de manera empírica probaron los conceptos o prototipos con personas cercanas. En el caso de sucesiones (empresas heredadas) no recurrieron a evaluación de producto, puesto que no hubo desarrollo o innovación. Tal como lo recomendó Steve Jobs: "ten el coraje para hacer lo que te dicen tu corazón y tu intuición" (Isaacson, 2011), todos los entrevistados interponen la intuición para su toma de decisiones, que, junto con una elevada autoconfianza y un comportamiento persistente, les permiten continuar luchando por su propósito (ver detalle en anexo 5).

Gráfico 4. Porcentaje de percepciones asociadas al tipo de aCtiviDAD UTILIZADA EN LOS PROCESOS DE PLANEACIÓN Y COMERCIALIZACIÓN

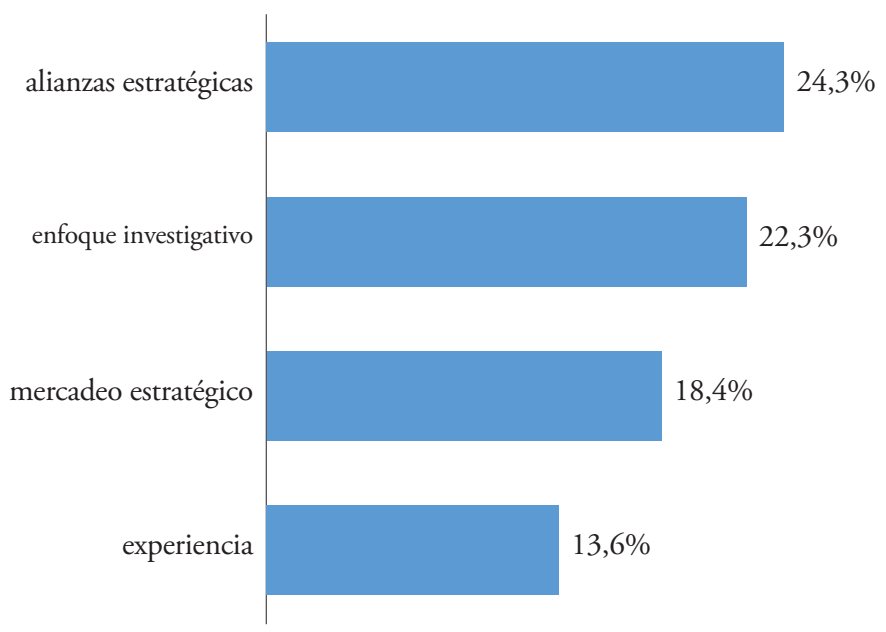

Fuente: elaboración de los autores, el valor porcentual es obtenido sobre el total de la muestra. 
Este comportamiento se explica, en parte, por la formación de cada emprendedor, existiendo una tendencia a desarrollar investigación o modelos predictivos por aquellos emprendedores con formación de maestría. Por otro lado, los emprendedores contactados de empresas medianas desarrollan procesos de planeación formal, en tanto, los de empresas pequeñas no recurren a ello. Lo que se puede asegurar también, es que los emprendedores entrevistados consideran que la formación es un elemento que juega un papel muy importante en el proceso de emprendimiento, tanto para los que ejercen su profesión de la que se graduaron como para aquellos que tomaron cursos, posgrados o cristalizaron sus ideas apoyados en un consultor. Para los emprendedores consultados es vital que la formación y experiencia en el negocio trascienda en sus colaboradores o en sus descendientes, en quienes esperan que más adelante tomen el control de sus compañías. Aquellos que estudiaron maestría son conscientes de la importancia del diseńo y desarrollo de un plan de negocio estructurado, así no lo hubieran implementado antes de incursionar en sus iniciativas emprendedoras; por ello, exhortaron a la población de nuevos emprendedores a estudiar, a formarse preferiblemente en maestría en administración, demostrando la eficacia de este modelo de formación.

Al comparar los anteriores resultados con los obtenidos en otras investigaciones recientes, se encuentra que si bien las pequeñas y medianas empresas (PYMES) de mayor rendimiento otorgan una mayor prioridad al marketing que a otras funciones comerciales, todavía están orientadas a la producción o la venta. Las PYMES de mayor rendimiento conocen mejor las herramientas de planificación estratégica. Los hallazgos sugieren que los principios de comercialización amplios y de pequeñas empresas contribuyen en cierta medida al éxito de las PYMES (Scheers y Makhitha, 2016). La tasa de fracaso de las pequeñas empresas es del $50 \%$ durante el primer año, pero se puede evitar si se implementa un plan estratégico efectivo (Rusell, 2018). La planificación estratégica de marketing es la columna vertebral para el éxito de las pequeñas empresas. Si bien el marketing estratégico tradicional se asociaba con las grandes empresas, las pequeñas empresas también deben emplear la planificación estratégica del marketing para sobrevivir ${ }^{c}$. Debido a la intensidad 
de la competencia, el cambio de mercado y los requisitos de los clientes, las pequeñas empresas deben adaptar sus actividades de marketing para reflejar estos cambios (Scheers y Makhitha, 2016). Las pequeñas empresas compiten en un entorno de naturaleza y alcance global, rica en información y basada en el conocimiento, que continúan influyendo en la forma en que operan (Abimbola y Kokac, 2007).

\section{Pensamiento efectual en emprendedores dominicanos}

Al comparar la información obtenida en la presente investigación, el comportamiento de los emprendedores dominicanos entrevistados guarda relación con el principio de pensamiento efectual denominado por Sarasvathy como "piloto en avión", el cual considera "depender y trabajar con el talento humano como el principal conductor de oportunidades" (Sarasvathy, 2003, p. 20).

En el otro principio denominado como Limonada, definido como "reconocer y apropiarse de la contingencia mediante las sorpresas en lugar de tratar de evitarlas, superarlas o adaptarlas" (Sarasvathy, 2003, p. 20), se fundamentan algunos de los emprendedores dominicanos, quienes tienden a esperar la presencia de contingencias que les lleva a tomar decisiones rápidas para superar el momento, sin dejar que se pierda su entusiasmo y mucho menos su idea emprendedora.

De manera adicional, un tercer principio que guarda relación con una proporción de los entrevistados fue el de "pájaro en mano", el cual plantea "negociar con todas las partes interesadas que estén dispuestas a asumir compromisos reales con el proyecto, sin preocuparse por los costos de oportunidad ni llevar a cabo análisis competitivos elaborados" (Sarasvathy, 2003, p. 20), los emprendedores dominicanos que iniciaron sus negocios partiendo de una idea propia, apalancaron la implementación en personas muy cercanas, logrando su participación activa en el nuevo negocio, con apoyo financiero o en procesos de prospección (ver detalle en anexo 6).

Los hallazgos anteriores son equivalentes a la conclusión principal de la investigación desarrollada por Vorontsova (2016), quien sugiere que "los emprendedores no utilizan de manera única ni la lógica causal ni la efectual. La mayoría de las veces, prefieren un modelo híbrido que facilite la 
toma de decisiones empresariales a través de la combinación de métodos y prácticas causales y efectuales. Además, esta combinación no es estable y varía con el tiempo de acuerdo con los desafíos de las principales empresas emergentes en diferentes etapas" (Voronstova, 2016, p. 75).

El gráfico 5 describe los diferentes principios de pensamiento efectual que guardan relación con el comportamiento de los emprendedores dominicanos, donde se puede observar que el "piloto en avión” es aquel que mayor incidencia presenta.

Gráfico 5. Principios de Pensamiento efectual QUe Caracterizan EL EMPRENDIMIENTO DOMINICANO

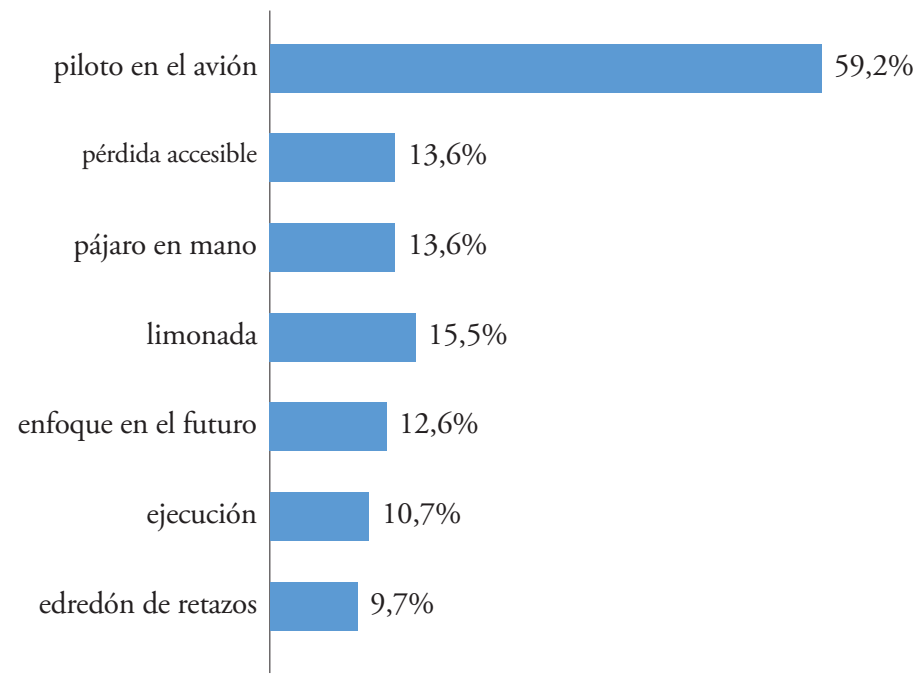

Fuente: elaboración de los autores, el valor porcentual es obtenido sobre el total de la muestra (99 entrevistas).

\section{Conclusiones}

Los dos rasgos de personalidad que guardan similitud en los emprendedores dominicanos consultados son la pasión y la aceptación del riesgo. Como competencias relevantes se encuentran las involucradas en la Orientación Emprendedora (Anlesinya y Bonuedi, 2015), que se componen de los factores de creatividad (incluye la generación de ideas) y la capacidad para percibir oportunidades (proactividad). Estas competencias guardan 
congruencia con los hallazgos de la reciente medición del Monitor Global de Emprendimiento - GEM (Barna Management School y Ministerio de Industria, Comercio y Mipymes, 2019), donde se encontró que el $75 \%$ de los dominicanos se consideran con las suficientes competencias ${ }^{\mathrm{d}}$ para iniciar un nuevo negocio, este valor es el mayor frente a los países de Latinoamérica y Caribe y el segundo frente a los otros 50 países participantes. Igualmente, tan solo el $12 \%$ de los emprendedores dominicanos entrevistados percibieron miedo al fracaso de emprender, el nivel más bajo de todos los países evaluados. Dentro de las competencias conceptuales y de relación predomina la persistencia, hasta el punto de que muchos de ellos se autocalifican como tercos.

Sin embargo, tan solo una parte de los emprendedores entrevistados fueron identificados con algunas competencias operacionales y contextuales, ya que la mayoría no desarrolla investigación de mercados previa a los procesos de innovación, como tampoco diseñan un plan de negocio estructurado. Los emprendedores con formación en maestría y aquellos que han fundado empresas, que en la actualidad se consideran medianas o grandes en tamaño, son quienes elaboran un plan de negocio, así como basan sus decisiones de innovación en la intuición, con posterior desarrollo de investigación de mercados. Estos procesos requieren de información actualizada del sector donde compiten en la República Dominicana, la cual no se encuentra disponible y mucho menos organizada. Lo anterior guarda una estrecha relación con el informe presentado por la Comisión Económica para América Latina y el Caribe (Godínez, 2009), quienes, desde hace 10 años han recomendado programas integrales y sistémicos de reforma de las políticas públicas. Su informe sugirió enérgicamente cambios estructurales hacia una producción más intensiva en tecnología y conocimiento para aumentar la eficiencia y la calidad, así como para agregar más valor a la producción nacional. Igualmente, la reciente información del Monitor Global de Emprendimiento, demuestra que la República Dominicana adolece actualmente de un entorno emprendedor adecuado, para que las iniciativas de aquellos que arriesgan su capital en nuevos emprendimientos logren un exitoso resultado (ver detalle en anexo 7). En este reporte se muestra que las políticas del gobierno y sus programas de

\footnotetext{
${ }^{\mathrm{d} C o n o c i m i e n t o s, ~ h a b i l i d a d e s ~ y ~ e x p e r i e n c i a . ~}$
} 
respaldo, así como el nivel de transferencia de investigación y desarrollo en el país son insuficientes para permitir una mayor competitividad de las nuevas empresas (Barna Management School y Ministerio de Industria, Comercio y Mipymes, 2019).

El comportamiento de los emprendedores dominicanos entrevistados se caracteriza por presentar, en mayor medida, uno de los principios de pensamiento efectual propuesto por Saras Sarasvathy: "piloto en avión", el cual se describe abreviadamente como "compromiso a capitalizar el talento humano que los rodea". En segunda instancia, una parte de los emprendedores guarda relación con los principios de "limonada", y "pájaro en mano", los cuales se refieren a aprovechar la contingencia en lugar de evitarla, y lograr el compromiso de los diferentes actores en el proceso de emprendimiento.

\section{Recomendaciones}

Para ofrecerle a la población de nuevos emprendedores dominicanos un ecosistema emprendedor adecuado, que facilite sus procesos de generar empresa, empleos y, por ende, continuar con su aporte al crecimiento económico del país, se requiere el diseño y desarrollo de modelos de enseñanza y transferencia de conocimiento, desde la educación primaria, hasta la educación superior a nivel de investigación y desarrollo.

En primera instancia, tal como lo corroboraron algunos estudios (Tsakiridou y Stergiou, 2015), y de acuerdo con los resultados del último reporte del Global Entrepreneurship Monitor (Barna Management School y Ministerio de Industria, Comercio y Mipymes, 2019), en la República Dominicana existe una gran oportunidad de implementar en la educación primaria y secundaria asignaturas con contenidos que mejoren las competencias emprendedoras de los estudiantes, pues en este factor de entorno emprendedor, en la medición del 2018, el país obtuvo una calificación por debajo del promedio de los demás países de la región y del mundo (ver detalles en anexo 8).

Igualmente, el sector académico de educación superior puede permanecer ofreciendo programas de postgrado que incluyan asignaturas de emprendimiento; rediseñando o implementando currículos en diferentes carreras que contemplen asignaturas transversales de emprendimiento — recomendación validada en una investigación con estudiantes 
(Martínez-Caro y Cegarra-Navarro, 2012)—. La medición de la eficacia de estas materias se puede desarrollar a través del inventario de competencias (Bendassolli, Borges-Andrade, Gondim y Makhamed, 2017), comparando los resultados en un pre-test, frente a un post-test o por medio de diversos modelos de investigación recientemente validados (Draksler y Sirec, 2018).

Para corroborar lo anteriormente expuesto, el privilegiado lugar competitivo que ha logrado Portugal en los últimos años ha sido por el diseño e implementación de un entorno emprendedor, donde participa activamente el gobierno. Dentro de sus políticas consideran clave capacitar a los fundadores y empleados del futuro para desarrollar habilidades empresariales. El ecosistema de inicio (start-up) de negocios en Portugal se beneficia de fundadores altamente educados, un gran número de instituciones de educación superior de alto rango, y se clasifica particularmente bien en términos de acceso al talento. La educación en emprendimiento ya comienza a nivel escolar en Portugal, con ocho escuelas diferentes que utilizan métodos y herramientas de la Escuela Emprendedora para el aprendizaje empresarial (Cuppen, 2018).

En segundo lugar, y teniendo en cuenta la dificultad de contar con información empresarial actualizada en la República Dominicana que permita identificar y cuantificar oportunidades de emprendimiento en el país, se recomienda generar una política, con sus actividades y estructura orgánica-funcional, que promueva la generación de conocimiento, así como la disponibilidad de información actualizada de desempeño de los diferentes sectores y sub-sectores, reduciendo así la brecha de conocimiento existente entre las PYMES y las grandes compañías dominicanas. Esta iniciativa podría incluir un modelo de consulta con ejes informativos tales como:

Actividad empresarial, que contenga información sobre el número de compañías en cada actividad económica, clasificación por tamaño y tipo de empresa, promedio de vida empresarial, constitución y cancelación de negocios, entre otros (Universidad Javeriana, 2019). Benchmarking empresarial, esto es, una herramienta vital para inversionistas, empresarios y emprendedores, que permite comparar sectores económicos, conocer sus empresas integrantes y clasificarlas según diferentes variables financieras. 
Reporte empresarial, que permite conocer la información general, contable, financiera, jurídica, de contratación estatal y de comercio exterior de las empresas nacionales y las inscritas en las Cámaras de Comercio de cada ciudad del país.

Investigaciones y papers obtenidos a partir de la articulación con las revistas que promueve el sector académico dominicano, incluido el INTEC.

La data se podría organizar en una plataforma dinámica, de consulta libre para los sectores académico y emprendedor, coordinada a partir del Observatorio de Competitividad, organismo dependiente del Consejo Nacional de Competitividad (CNC), teniendo como fuentes de información los reportes financieros periódicos entregados por las empresas de cada sector al Ministerio de Hacienda de la República Dominicana.

De manera adicional, esta herramienta permitiría que las PYMES accedan a un modelo de planeación estratégica, la cual exige información actualizada del entorno, incluyendo el sector económico donde se participa con el nuevo negocio, data que actualmente adolece la República Dominicana. Lo anterior se valida por medio de los resultados de una investigación con 254 PYMES dominicanas (Aristy, 2012), que encontró que la actual política de apoyo gubernamental directo no ejerce una influencia estadísticamente significativa sobre la probabilidad de éxito de esas empresas. Entre las políticas a desarrollar, el autor propone aquellas que aumenten el conocimiento de la gestión empresarial, el cual sería un apoyo importante para los emprendedores, garantizando la creación de empresas orientadas hacia el mercado.

Futuras investigaciones en la República Dominicana deberían considerar el diseño y aplicación de un instrumento que, de manera pertinente, contenga las baterías de preguntas correspondientes a cuantificar las competencias emprendedoras y rasgos de personalidad tenidos en cuenta en la presente investigación, así como los referidos de la literatura mencionada. Por otro lado, al aplicar una herramienta adecuada, podrían inferir si las decisiones de inversión iniciales en las nuevas empresas, los emprendedores aplican la lógica de efectuación. De esta forma se podría corroborar la hipótesis de Vorontsova (2016), la cual menciona que "el principio de pérdida asequible junto con su examen de medios, se percibieron como el mejor razonamiento posible para tomar decisiones sobre 
su primera inversión" (p. 75). Así también, se podrían identificar los principios (no solamente del modelo efectual) que van siendo utilizados durante el ciclo de vida de la empresa.

\section{Referencias}

Abimbola, T. \& Kocak, A. (2007). "Brand, organizational identity and reputation: SMEs as expressive organizations, a resource based perspective", International Journal of Qualitative Market Research, 10(4), 416-430. doi:10.1108/13522750710819748

Anlesinya, A. \& Bonuedi. A. (2015). Entrepreneurial orientation and profitability nexus: evidence from micro enterprises in the retail sector in Madina-Accra. Conference paper. Disponible en: https:// www.researchgate.net/publication/288834047_Anlesinya_A_ Bonuedi_A_A_2015_Entrepreneurial_orientation_and_profitability_nexus_evidence_from_micro_enterprises_in_the_retail_ sector_in_Madina-Accra

Aparicio, S., Urbano, D. \& Gómez, D. (2018). Entrepreneurship and regional economic growth in Antioquia: An empirical analysis. Economics and Business Letters. 7. doi: 10.17811/ebl.7.2.2018.84-92. https:/www.researchgate.net/publication/326270964_Entrepreneurship_and_regional_economic_growth_in_Antioquia_An_ empirical_analysis

Aristy, J. (2012). Determinantes del éxito de las PYMES en la República Dominicana. Ciencia y Sociedad, 37(4): 497-528. Disponible en http://finanzasfae.uprrp.edu/wp-content/uploads/2015/10/ Mun\%CC\%83oz-Longobardi-2012.pdf

Barna Management School y Ministerio de Industria, Comercio y Mipymes. (2019). GEM República Dominicana 2018. ISBN: 978-99458-0578-9.

Barron, F. \& Harrington, D. (1981). Creativity, intelligence, and personality. Annual review of psychology. 1981. Recuperado de https:// pdfs.semanticscholar.org/faa7/a22aac37c00726237696dc423b4f412a53ed.pdf 
Bendassolli, P., Borges-Andrade, J., Gondim, S. \& Makhamed, J. (2017). Performance, self-regulation, and competencies of entrepreneurs in Brazilian creative industries. Psicologia: Teoria e Pesquisa. 32 no.spe Brasília 2016 Epub Mar 27, 2017. doi: http://dx.doi. org/10.1590/0102-3772e32ne221. Print version ISSN 0102-3772. On-line version ISSN 1806-3446

Blankson, C. \& Stokes, D., (2002). Marketing practices in the UK small business sector. Marketing Intelligence \& Planning, 20(1), 49-61. Recuperado de https://bdbib.javerianacali.edu.co:2519/docview/ $213112177 / 8695$ F992540941F2PQ/ ? accountid $=13250$

Brandstätter, H. (2010). Personality aspects of entrepreneurship: A look at five meta-analyses. Johannes-Kepler-University, Social and Economic Psychology Unit, Altenberger. Science Direct, 201007007. 222-230. Recuperado de https://www.academia.edu/36527465/ Personality_aspects_of_entrepreneurship_A_look_at_five_ meta-analyses

Bruns, K., Bosma, N., Sanders, M. \& Schramm, M. (2017). Searching for the existence of entrepreneurial ecosystems: a regional cross-section growth regression approach. Small Bus Econ (2017) 49: 31-54. doi: $10.1007 /$ s1 1187-017-9866-6

Cagica, L. (2017). Entrepreneurial Ecosystems. In book: Handbook of Research on Entrepreneurial Development and Innovation Within Smart Cities. doi: 10.4018/978-1-5225-1978-2.ch001

CEPAL. (2010). Un Índice Regional de Competitividad para un país. Revista CEPAL (102). Diciembre 2010. Recuperado de https:// www.cepal.org/es/publicaciones/37386-revista-cepal-no102

Cruz-Ros, S., Mas-Tur, A. \& Garzón, G. (2017). Psychology and Marketing 34(11):1031-1038 October 2017. doi: 10.1002/mar.21042

Cuppen, J. (2018). An overview of the Portuguese Entrepreneurship Ecosystem. Halbe and Koenraads. Recuperado de https://www. halbekoenraads.com/wp-content/uploads/2018/06/BOOKLET_ PORTUGAL.small_.pdf

Davis, M., Hall, J. \& Mayer, P. (2015). Developing a new measure of entrepreneurial mindset: Reliability, validity, and implications for practitioners. Consulting Psychology Journal: Practice and Research. 68(1), 21-48. doi: 10.1037/cpb0000045 
Draksler, T. \& Sirec, K. (2018). Conceptual Research Model for Studying Students' Entrepreneurial Competencies. Naše gospodarstvol Our economy. 64. 23-33. doi: 10.2478/ngoe-2018-0020

Ministerio de Industria, Comercio y Mipymes. (2016). Presentación / introducción - Ley no. 688-16 de emprendimiento y decreto 160-18 reglamento del fondo Confie. Recuperado de https://micm.gob.do/ images/pdf/otros/Intro-Ley-688-16-y-Decreto-160-18.pdf

The Global Entrepreneurship and Development Institute (2018). The Global Entrepreneurship Index 2018. Washington, D.C., EE. UU. Recuperado de https://thegedi.org/global-entrepreneurship-and-development-index/

George, J. \& Zhou, J. (2001). When openness to experience and conscientiousness are related to creative behavior: an interactional approach. Journal of Applied Psychology, 86, 513-524. doi:10.1037/00219010.86.3.513

Godínez, V. \& Máttar, J. (2009). La República Dominicana en 2030: Hacia una nación cohesionada. Serie: Libros de la CEPAL. No. 104, 582 p. Recuperado de https://www.cepal.org/es/publicaciones/2563-la-republica-dominicana-2030-nacion-cohesionada.

Hamel, G. (2000). Leading the IT revolution. Harvard Business School Press. 22(12). Part 1, December 2000. doi: 10.12691/jbms-3-2-4

Hill, J., (2001). A multidimensional study of the key determinants of effective SME marketing: Part 1. International Journal of Entrepreneurship Behaviour \& Research, 7(5), 171-204.

Hills, G. \& Hultman, C. (2006). Entrepreneurial Marketing. S. Lagrosen G. \& Svensson (Eds.) Marketing: Broadening the Horizons. Lund: Studentlitteratur. pp. 220-234.

Isaacson, Walter. (2011). Biography of Steve Jobs. New York, EE. UU.: Simon and Schuster Inc.

Martínez-Caro, E. y Cegarra-Navarro, J. (2012). El desarrollo de competencias transversales mediante proyectos de emprendimiento en el marco de una asignatura de dirección de operaciones. Polipapers, Working Papers on Operations Management, 3(2), 9-13. Recuperado de https://polipapers.upv.es/index.php/WPOM/article/view/978

Mason, C. \& Brown, R. (2014). Entrepreneurial ecosystems and growth oriented entrepreneurship. Background paper prepared for the workshop organised by the OECD LEED. Programme and the 
Dutch Ministry of Economic Affairs on Entrepreneurial Ecosystems and Growth Oriented Entrepreneurship. The Hague, Netherlands, [7th November 2013]. p. 3-5. Recuperado de https://www.oecd. org/cfe/leed/entrepreneurial-ecosystems.pdf

Monllor, J. \& Altay, N. (2016). Discovering opportunities in necessity: The inverse creative destruction effect. Journal of Small Business and Enterprise Development, 23(1), 274-291. Recuperado de https:// www.academia.edu/22452878/Discovering_opportunities_in_ necessity_the_inverse_creative_destruction_effect

Morrish, S., Deacon, J. \& Miles, M. (2010). Entrepreneurial marketing: acknowledging the entrepreneur and customer-centric interrelationship. JournalofStrategic Marketing, 18(4), 303-316. Recuperado de https://www.researchgate.net/publication/233258555_Entrepreneurial_marketing_Acknowledging_the_entrepreneur_and_ customer-centric_interrelationship

Organización para la Cooperación y el Desarrollo Económicos, Corporación Andina de Fomento y Comisión Económica para América Latina y el Caribe (2018). Perspectivas económicas de América Latina 2018: Repensando las instituciones para el desarrollo. Éditions OCDE, París. doi: 10.1787/leo-2018-es

Organisation for Economic Co-operation and Development. Developing entrepreneurship competencies. (2018). SME Ministerial Conference. 22-23 February 2018. Mexico City. Recuperado de https:// www.oecd.org/cfe/smes/ministerial/documents/2018-SME-Ministerial-Conference-Parallel-Session-3.pdf

Pekkala, S. 2017. Personality Traits of Entrepreneurs: A Review of Recent Literature. Harvard Business School. Working Paper 18-047. Recuperado de https://www.hbs.edu/faculty/Publication\%20Files/18047_b0074a64-5428-479b-8c83-16f2a0e97eb6.pdf

Rauch, A. and Frese, M. (2007). Born to be an entrepreneur? Revisiting the personality approach to entrepreneurship. In J.R. Baum, M. Frese, \& R.A. Baron (Eds.), The psychology of entrepreneurship. January 2007. 41-65. Recuperado de https://www.researchgate.net/ publication/270820381_Born_to_Be_an_Entrepreneur_Revisiting_the_Personality_Approach_to_Entrepreneurship 
Reynolds, P. (2005). Entrepreneurship Research Innovator, Coordinator, and Disseminator. Small Business Economics 24(4), 351-358. Recuperado de https://www.researchgate.net/publication/5158285_Paul_D_ Reynolds_Entrepreneurship_Research_Innovator_Coordinator_ and_Disseminator

Rusell, L. (2018). A Review of the Future State of Strategic Planning for Small Business Owners. Proquest number: 10828033. Recuperado de https://bdbib.javerianacali.edu.co:2519/docview/2056464464/ BD69FE9354C14ABFPQ/15? accountid $=13250$

Sarasvathy, S. (2003). Effectuation: elements of entrepreneurial expertise. Recuperado de https://www.researchgate.net/publication/228786046_ Effectuation_Elements_of_Entrepreneurial_Expertise

Sarasvathy, S. (2008). University of Virginia marketing under uncertainty: the logic of an effectual approach. Journal of marketing, 73, may 2009. Recuperado de https://www.effectuation.org/wp-content/ uploads/2017/06/Marketing-under-Uncertainty.pdf

Sarasvathy, S. \& Kotha, S. (2001). Effectuation in the management of knightian uncertainty: evidence from the real networks case. University of Washington Business School. Seattle, WA. Mackenzie Hall. Recuperado de https://www.effectuation.org/wp-content/ uploads/2016/06/2001-realnet-1.pdf

Schmitt-Rodermund, E. (2003). Pathways to Successful Entrepreneurship: Parenting, Personality, Early Entrepreneurial Competence, and Interests. Journal of Vocational Behavior 65(3), 498-518. December 2004. Recuperado de https://www.researchgate.net/ publication/223410024_Pathways_to_Successful_Entrepreneurship_Parenting_Personality_Early_Entrepreneurial_Competence_ and_Interests

Seibert, S., Kraimer, M., \& Crant, J. (2001). What do proactive people do? A longitudinal model linking proactive personality and career success. Personnel Psychology, 54, 845-874.

Siu, W. (2000). Marketing and company performance of Chinese small firms in Hong Kong. Marketing intelligence \& planning, 18(5), 292-307.

Siu, W., Fang, W., \& Lin, T., (2004). Strategic marketing practices and the performable of small and medium-sized enterprises (SMEs) in 
Taiwan. Entrepreneurship \& Regional Development: An International Journal, 16(2), 61-178.

The World Bank. (2018). New business registered. www.data.worldbank.org Tiago, T., Faria, S. \& Couto, J. P. (2015). Fostering innovation by promoting entrepreneurship: From education to intention. Procedia-Social and Behavioral Sciences, 175, 154-161. Recuperado de https://www.researchgate.net/publication/273142067_Fostering Innovation_by_Promoting_Entrepreneurship_From_Education_ to_Intention

Tsakiridou, H. and Stergiou, K. (2015). Entrepreneurship Competences and Entrepreneurial Intentions of students in Primary Education. Journal of Humanities, Social Sciences and Education (JHSSE), 1, 106-117. Recuperado de https:/www.researchgate.net/publication/ 280920902_Entrepreneurship_Competences_and_Entrepreneurial_Intentions_of_students_in_Primary_Education

Universidad Javeriana Cali. (2019). Recursos digitales. Recuperado de https://www.javerianacali.edu.co/biblioteca/recursos-digitales/ fuera-del-campus

Van Scheers, L. and Makhitha, K. (2016). Are Small and Medium Enterprises (SMEs) Planning for Strategic Marketing in South Africa? Foundations of Management. 8. doi: 10.1515/fman-2016-0019

Veciana, J. \& Urbano, D. 2008. The institutional approach to entrepreneurship research. Introduction. International Entrepreneurship and Management Journal, 4(4), 365-379 .75-377. Recuperado de https:/www.researchgate.net/publication/226016905_The_institutional_approach_to_entrepreneurship_research_Introduction

Viinikainen, J., Heineck, G., Böckerman, P., Hintsanen, M., Raitakari, O., Pehkonen, J. (2016). Born Entrepreneur? Adolescents' Personality Characteristics and Self-Employment in Adulthood. Discussion paper series. Forschungsinstitut zur Zukunft der Arbeit Institute for the Study of Labor. Recuperado de http://ftp.iza.org/dp9805.pdf

Vorontsova, O. (2016). The analysis of the impact of Causation and Effectuation approaches on decision-making of IT start-ups, (dissertation of Master in Management). U. Porto. Economia e gestao. Recuperado de https://www.effectuation.org/wp-content/uploads/2017/06/Theanalysis-of-the-impact-of-Causation-and-Effectuation-approacheson-decision-making-of-IT-start-ups-1.pdf 
Welter, F. \& Smallbone, D. (2011). Institutional Perspectives on Entrepreneurship. Handbook of Organisational Entrepreneurship, Publisher: Elgar, Editors: Daniel Hjorth, pp.64-78.

World Intelectual Property Organization (WIPO). (2018). World Innovation Index 2018, 249. Recuperado de https:/www.wipo.int/edocs/ pubdocs/en/wipo_pub_gii_2018.pdf

World Economic Forum. (2018). The Global Competitiveness Report 2018. 106. Recuperado de http://www3.weforum.org/docs/ GCR2017-2018/05FullReport/TheGlobalCompetitivenessReport 2017\%E2\%80\%932018.pdf

Zdolšek D. \& Sirec, K. (2018). Conceptual Research Model for Studying Students' Entrepreneurial Competencies. Naše gospodarstvo/Our economy, 64, 23-33. doi: 10.2478/ngoe-2018-0020 


\section{Anexos}

Anexo 1. Procedimiento de anÁlisis de la información de las 99 ENTREVISTAS EN ATLAS.TI

Para el análisis cualitativo de las entrevistas con ATLAS.TI se almacenaron las 99 entrevistas o documentos primarios en la Unidad Hermenéutica, con sus respectivos códigos — son las categorías o factores de análisis de las dimensiones_- anotaciones o memos — surgen de anotaciones que se considera importante tener en cuenta_, las familias de documentos primarios, las familias de códigos y familias de memos:

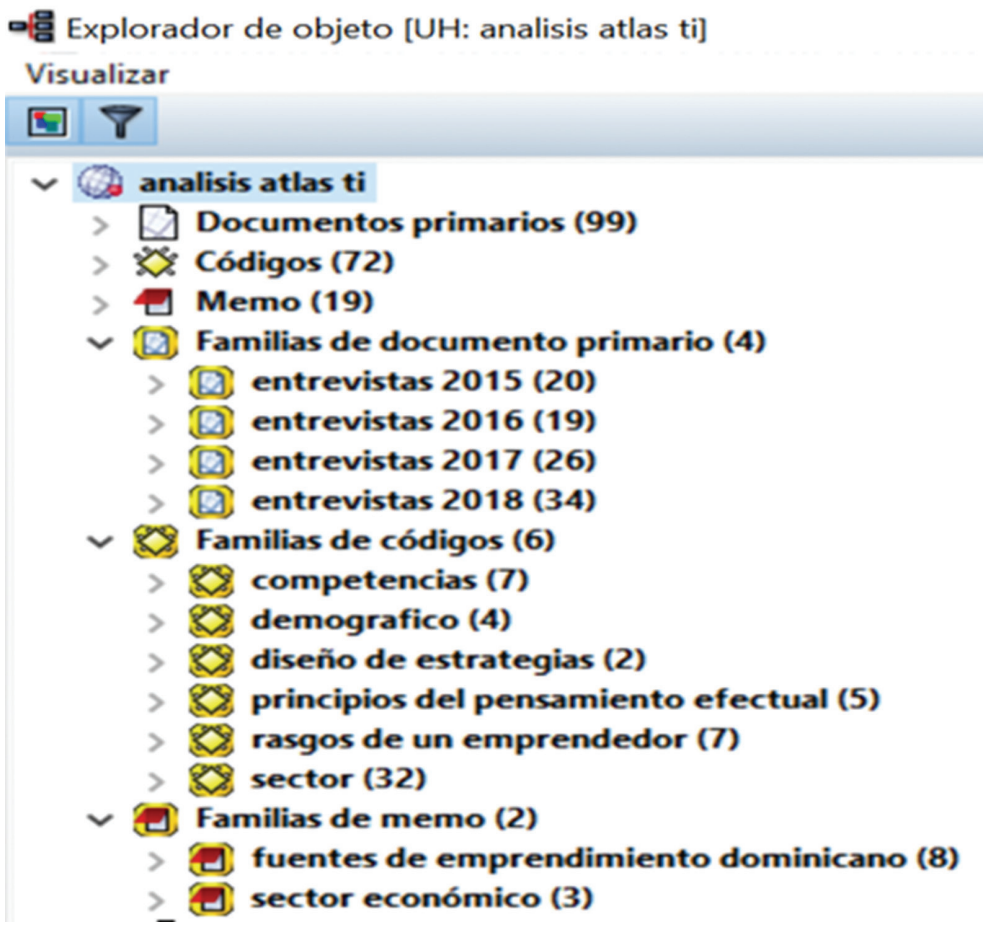

Fuente: esquema de salida del paquete Atlas.ti, elaboración de los autores. 


\section{Anexo 2. Perfil de los entrevistados}

\section{Demográficos}

2. Administrador de códigos [UH: analisis atlas ti]

Códigos Edición Miscelánea Imprimir Visualizar

\begin{tabular}{|c|c|c|c|c|c|c|c|}
\hline 当 $\odot \Leftrightarrow \varnothing$ 啳 & $x$ & 口回 一 & (a) $B$ & iscar & \multicolumn{3}{|c|}{$x$} \\
\hline Nombre & & Fundamentado & De... & Autor & Creado & Modifica... & Familias \\
\hline Z experiencia & a & 14 & 0 & Super & $08 / 11 / 20 \ldots$ & $08 / 12 / 20 \ldots$ & demografico \\
\hline formacion & E & 61 & 0 & Super & $08 / 11 / 20 \ldots$ & $07 / 12 / 20 \ldots$ & demografico \\
\hline 2 hombre & & 83 & 0 & Super & $08 / 11 / 20 \ldots$ & $07 / 12 / 20 \ldots$ & demografico \\
\hline mujer & 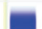 & 16 & 0 & Super & $08 / 11 / 20 \ldots$ & $07 / 12 / 20 \ldots$ & demografico \\
\hline
\end{tabular}

Fuente: esquema de salida del paquete Atlas.ti, elaboración de los autores.

Sector de la empresa

2. Administrador de códigos [UH: analisis atlas ti]

Códigos Edición Miscelánea Imprimir Visualizar

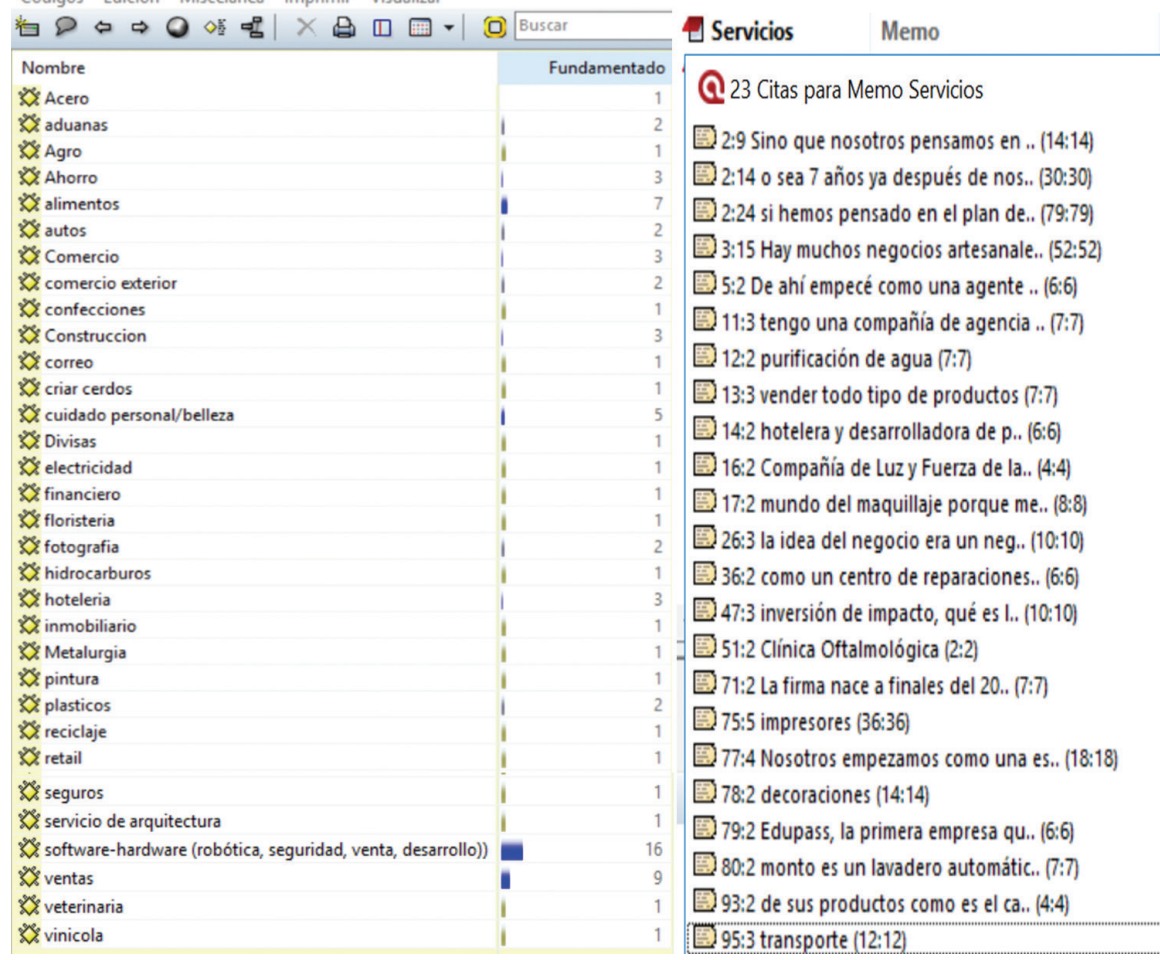

Fuente: esquema de salida del paquete Atlas.ti, elaboración de los autores. 
Anexo 3. Fundamentación de los Rasgos de PERSONALIdAd IDENTIFiCADOS EN LAS 99 ENTREVISTAS, PROCESADO POR MEDIO DE ATLAS.TI

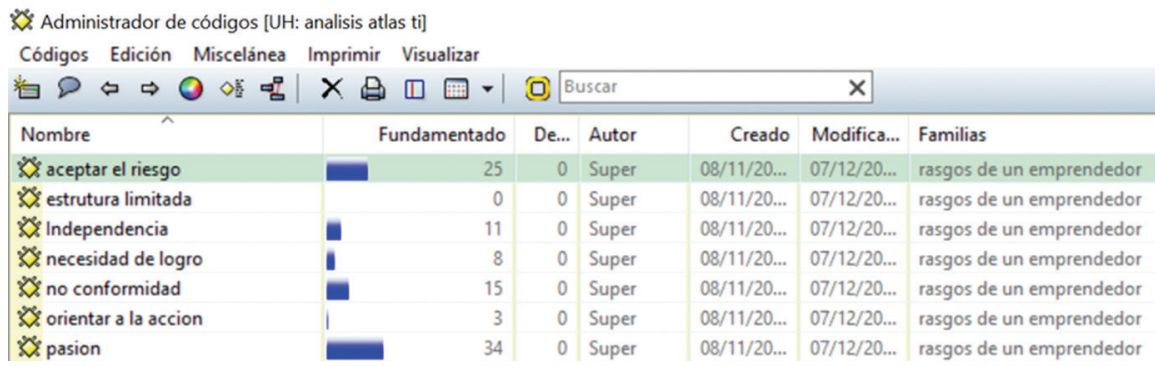

Fuente: esquema de salida del paquete Atlas.ti, elaboración de los autores.

ANEXo 4. FundaMENTACIÓN DE LAS COMPETENCIAS DE ORIENTACIÓN
EMPRENDEDORA, CONCEPTUALES Y DE RELACIÓN IDENTIFICADAS EN LAS 99 ENTREVISTAS, PROCESADO POR MEdio dE AtLAS.TI

Administrador de códigos [UH: analisis atlas ti]

Códigos Edición Miscelánea Imprimir Visualizar

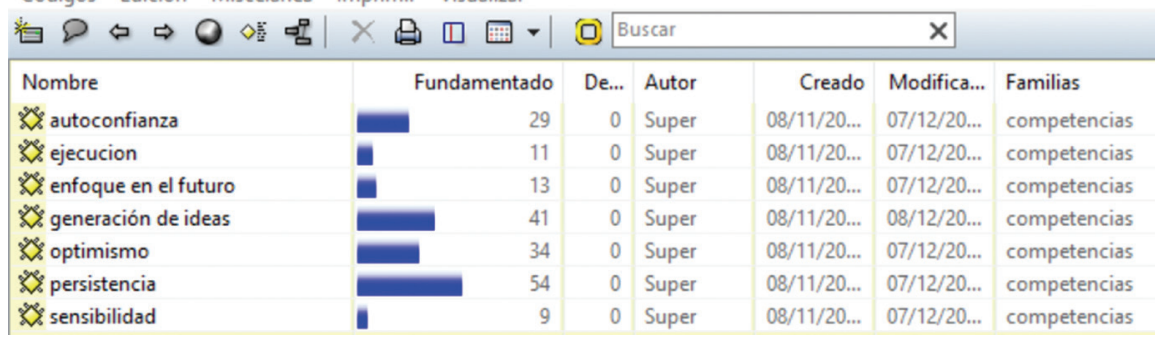

Fuente: esquema de salida del paquete Atlas.ti, elaboración de los autores.

AneXo 5. Fundamentación de las COMPETENCIAS OPERACIONALES Y CONTEXTUALES IDENTIFICADAS EN LAS 99 ENTREVISTAS, PROCESADO POR MEDio dE ATLAS.TI

\begin{tabular}{|c|c|c|c|c|c|c|c|}
\hline Códigos Edición Miscelánea & \multicolumn{2}{|c|}{ Imprimir Visualizar } & \multirow{2}{*}{\multicolumn{2}{|c|}{ (D) Buscar }} & & & \\
\hline 当 $\rho \Leftrightarrow \Rightarrow$ 嗼 & $\times[B$ & 四国・ & & & \multicolumn{2}{|r|}{$x$} & \\
\hline Nombre & & Fundamentado & De... & Autor & Creado & Modifica... & Familias \\
\hline \% investigativa & 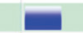 & 23 & 0 & Super & $08 / 11 / 20 \ldots$ & $08 / 12 / 20 \ldots$ & diseño de estrategias \\
\hline predictiva & & 37 & 0 & Super & $08 / 11 / 20 \ldots$ & $08 / 12 / 20 \ldots$ & diseño de estrategias \\
\hline
\end{tabular}

Fuente: esquema de salida del paquete Atlas.ti, elaboración de los autores. 
Anexo 6. Principios de pensamiento efectual encontrados en las 99 entrevistas, procesado por medio de Atlas.ti

\begin{tabular}{|c|c|c|c|c|c|c|c|}
\hline Códigos Edición Miscelánea & Imprimir & Visualizar & & & & & \\
\hline 当 $P \Leftrightarrow \Rightarrow 0$ 湢 & $\times \Leftrightarrow$ & 묘 & | & Buscar & & $x$ & \\
\hline Nombre & & damen... & De... & Autor & Creado & Modifica... & Familias \\
\hline Edredon de retazos & I & 10 & 0 & Super & $08 / 11 / 20 \ldots$ & $07 / 12 / 20 \ldots$ & principios del pensamiento efect... \\
\hline 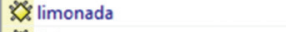 & 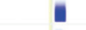 & 16 & 0 & Super & $08 / 11 / 20 \ldots$ & $07 / 12 / 20 \ldots$ & principios del pensamiento efect... \\
\hline 20. Pajaro en mano & 】 & 14 & 0 & Super & $08 / 11 / 20 \ldots$ & $07 / 12 / 20 \ldots$ & principios del pensamiento efect... \\
\hline 20 Perdida accesible & ע & 14 & 0 & Super & $08 / 11 / 20 \ldots$ & $07 / 12 / 20 \ldots$ & principios del pensamiento efect... \\
\hline W piloto en el avión & 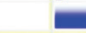 & 61 & 0 & Super & $08 / 11 / 20 \ldots$ & $07 / 12 / 20 \ldots$ & principios del pensamiento efect... \\
\hline
\end{tabular}

Fuente: esquema de salida del paquete Atlas.ti, elaboración de los autores.

ANeXo 7. Evolución de los FACTORES DEL ENTORNO EMPRENDEDOR EN la República Dominicana, 2008 FRENTE AL 2018

\begin{tabular}{|l|c|c|}
\hline Factores de entorno emprendedor & 2018 & 2008 \\
\hline Políticas gubernamentales: emprendimiento como prioridad y su apoyo & 2,14 & 2,06 \\
\hline Políticas gubernamentales: burocracia e impuestos & 2,30 & 2,62 \\
\hline Programas gubernamentales dirigidos al emprendimiento & 3,02 & 2,45 \\
\hline Educación y formación emprendedora etapa escolar & 1,52 & 1,73 \\
\hline Nivel de educación empresarial en el ámbito profesional & 3,08 & 3,17 \\
\hline Nivel de transferencia en Investigación y Desarrollo & 1,69 & 2,18 \\
\hline Existencia y acceso a infraestructura comercial y profesional & 2,58 & 2,77 \\
\hline Entorno financiero relacionado con el emprendimiento. & 1,71 & 1,84 \\
\hline Dinámica del mercado interno & 2,62 & 2,96 \\
\hline Barreras de acceso al mercado interno & 2,26 & 2,39 \\
\hline Existencia y acceso a infraestructura fisica y de servicios & 3,89 & 3,67 \\
\hline Normas culturales, sociales y de apoyo a la sociedad. & 3,37 & 3,21 \\
\hline
\end{tabular}

Fuente: Barna Management School y Ministerio de Industria, Comercio y Mipymes - GEM, 2019.

Anexo 8. Factores del entorno emprendedor en la República DOMINICANA FRENTE A OTROS PAÍSES, 2018

\begin{tabular}{|l|c|c|c|c|}
\hline Factores de entorno emprendedor & $\begin{array}{c}\text { República } \\
\text { Dominicana }\end{array}$ & OECD & $\begin{array}{c}\text { altos } \\
\text { ingresos }\end{array}$ & $\begin{array}{c}\text { Total países } \\
\text { GEM }\end{array}$ \\
\hline Políicas gubernamentales: emprendimiento como prioridad y su apoyo & 3,51 & 4,55 & 4,54 & 4,38 \\
\hline Políticas gubernamentales: burocracia e impuestos & 3,66 & 4,02 & 4,04 & 3,93 \\
\hline Programas gubernamentales dirigidos al emprendimiento & 5,04 & 5,15 & 5,07 & 4,75 \\
\hline Educación y formación emprendedora etapa escolar & 2,34 & 3,20 & 3,21 & 3,15 \\
\hline Nivel de educación empresarial en el ámbito profesional & 5,02 & 4,95 & 4,90 & 4,85 \\
\hline Nivel de transferencia en Investigación y Desarrollo & 2,63 & 4,19 & 4,17 & 3,98 \\
\hline Existencia y acceso a infraestructura comercial y profesional & 4,27 & 4,93 & 4,90 & 4,85 \\
\hline Entorno financiero relacionado con el emprendimiento. & 2,68 & 4,18 & 4,14 & 4,10 \\
\hline Dinámica del mercado interno & 4,36 & 4,60 & 4,62 & 4,85 \\
\hline Barreras de acceso al mercado interno & 3,62 & 4,22 & 4,23 & 4,15 \\
\hline Existencia y acceso a infraestructura fisica y de servicios & 6,58 & 6,58 & 6,53 & 6,37 \\
\hline Normas culturales, sociales y de apoyo a la sociedad. & 5,57 & 4,81 & 4,75 & 4,83 \\
\hline
\end{tabular}

Fuente: Barna Management School y Ministerio de Industria, Comercio y Mipymes - GEM, 2019. 